\title{
Correlations between center vortices and low-lying Dirac eigenmodes
}

\section{R. Höllwieser*}

Atomic Institute, University of Technology, Wiedner Hauptstr. 8-10, A-1040 Vienna, Austria

E-mail: hroman@kph.tuwien.ac.at

\section{Faber}

Atomic Institute, University of Technology, Wiedner Hauptstr. 8-10, A-1040 Vienna, Austria

E-mail: faber@kph.tuwien.ac.at

\section{J. Greensite}

Physics and Astronomy Dept., San Francisco State University, San Francisco, CA 94132, USA

E-mail: jgreensite@gmail.com

\section{Urs Heller}

American Physical Society, One Research Road, Box 9000, Ridge, NY 11961-9000, USA

E-mail: heller@aps.org

\section{Š. Olejník}

Institute of Physics, Slovak Academy of Sciences, SK-845 11 Bratislava, Slovakia

E-mail: stefan.olejnik@gmail.com

\begin{abstract}
Correlations between center vortices and low-lying eigenmodes of the Dirac operator are studied in both the overlap and asqtad formulations. In particular we suggest a solution to a puzzle raised some years ago by Gattnar et al. [Nucl. Phys. B 716 (2005) 105], who noted the absence of lowlying Dirac eigenmodes required for chiral symmetry breaking in center-projected configurations. We show that the low-lying modes are present in the staggered (asqtad) formulation, but not for overlap, and we argue that this is due to the absence of a field-independent chiral symmetry on the very rough center-projected configurations for overlap and "chirally improved" fermions. We also confirm and extend the results of Kovalenko et al. [Phys. Lett. B 648 (2007) 383]: there are strong correlations between center vortex locations and the scalar density of low-lying Dirac eigenmodes, supporting the picture of topological charge from center vortices.
\end{abstract}

8th Conference Quark Confinement and the Hadron Spectrum

September 1 - 6, 2008

Mainz, Germany

${ }^{*}$ Speaker. 


\section{Introduction}

The center vortex model explains quark confinement and the Casher argument [1] implies that a force strong enough to confine quarks is also generally expected to break chiral symmetry. The Banks-Casher relation [2] relates chiral symmetry breaking $(\chi S B)$ with a finite density of near-zero eigenmodes of the chiral-invariant Dirac operator. Several years ago, however, Gattnar et al. [3] reported a puzzling result with a chirally-improved version of the Dirac operator due to Gattringer [4]. They found a large gap around zero in the spectrum for center-projected configurations, which contain only thin vortex excitations and which are confining, implying zero chiral condensate and therefore no $\chi S B$. We suggest that this large gap found by Gattnar et al. is related to the way in which chiral symmetry is realized on the lattice. The Casher argument [1] is based on the usual $S U\left(N_{f}\right)_{L} \times S U\left(N_{f}\right)_{R}$ symmetry of the continuum theory with massless fermions. Center-projected configurations are, however, maximally discontinous; plaquette variables make a sudden transition from the trivial center element outside the thin vortex, to a non-trivial center element inside. The chirally-improved Dirac operator is not necessarily chirally symmetric, even approximately, in such backgrounds and there is no reason to expect spontaneous symmetry breaking.

We will reinforce these arguments in section 2, looking at the spectra of the overlap [5] and asqtad [6] Dirac operators, when evaluated on normal, vortex-only (i.e. center-projected), and vortex-removed lattices. Our results support the view that center vortices alone can induce both confinement and chiral symmetry breaking. In section 3 we report on other correlations between center-vortex locations and the density distribution of low-lying Dirac eigenmodes, following the earlier work by Kovalenko et al. [7]. These correlations support the picture advocated by Engelhardt and Reinhardt [8], in which topological charge is concentrated at points where vortices either intersect, or twist about themselves ("writhe") in a certain way.

Throughout this article we work with lattices generated by lattice Monte Carlo simulation of the tadpole improved Lüscher-Weisz pure-gauge action, mainly at coupling $\beta_{L W}=3.3$ (lattice spacing $a=0.15 \mathrm{fm}$ ) for the $S U(2)$ gauge group [9]. Center projection is performed in direct maximal center gauge (adjoint Landau gauge).

\section{Low-lying eigenmodes and thin vortices}

We present the first twenty overlap eigenvalues for a $16^{4}$ lattice at $\beta_{L W}=3.3$ in Fig. 1 . There is a big gap around zero for center-projected data, indicating zero chiral condensate. Looking closer at the center-projected eigenvalues one spots only five of the twenty eigenvalues. This indicates a degenerency of four, caused by the real trivial link variables $\left( \pm \mathbb{1}_{2}\right)$, where the two colors decouple and the eigenvalue equation $D \psi_{n}=\lambda_{n} \psi_{n}$ is invariant under charge conjugation. We speculated that the reason for the large gap in the vortex-only case was connected with the lack of smoothness of center-projected lattices. The chiral symmetry transformations are gauge-field dependent [10], and only approximate the $S U\left(N_{f}\right)_{L} \times S U\left(N_{f}\right)_{R}$ transformations of the continuum theory for configurations which vary slowly at the scale of the lattice spacing. Center-projected configurations are not even close to smooth, and the Casher argument, relating confinement to $\chi S B$ need not apply. However, the overlap operator should produce a more reasonable answer when applied to a smoother version of the center-projected lattice. Therefore we perform an interpolation 

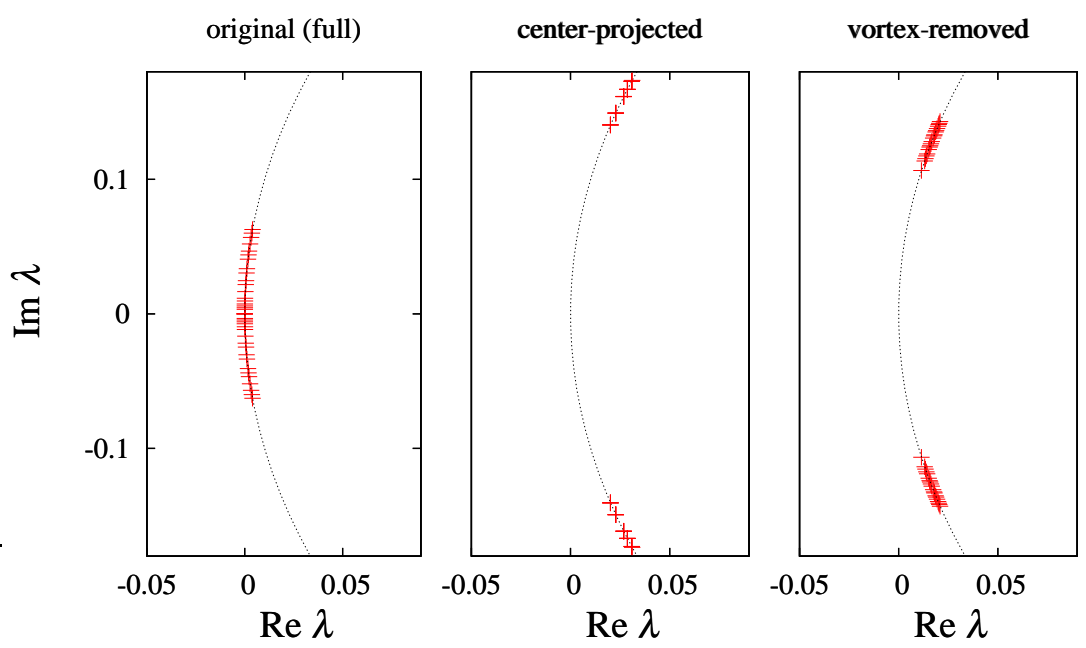

Figure 1: The first twenty overlap Dirac eigenvalue pairs on the Ginsparg-Wilson circle for a $16^{4}$ lattice at $\beta_{L W}=3.3$ for antiperiodic boundary conditions. The center-projected configurations show a four-fold degeneracy.

between full (gauged) and projected configurations, reducing the angle between the vector representing group element $U_{\mu}(x)$ in maximal center gauge, and the vector representing the $S U(2)$ center element $Z_{\mu}(x) I_{2}$ by some fixed percentage. In Fig. 2 we show the low-lying eigenvalues for partial projections together with the unprojected and center-projected lattices. We see that there is no really obvious gap in the partially-projected lattices, even at $85 \%$ projection. This agrees with our conjecture that applying the overlap operator to a smoother version of the vortex-only vacuum would give a result consistent with $\chi S B$ and the Banks-Casher relation. Staggered and asqtad fermions, on the other hand, do not require a smooth configuration to preserve a subgroup of the usual continuum $S U\left(N_{f}\right)_{L} \times S U\left(N_{f}\right)_{R}$ symmetry, and by the Casher argument [1] one would expect this remaining symmetry to be spontaneously broken by any confining gauge configuration.

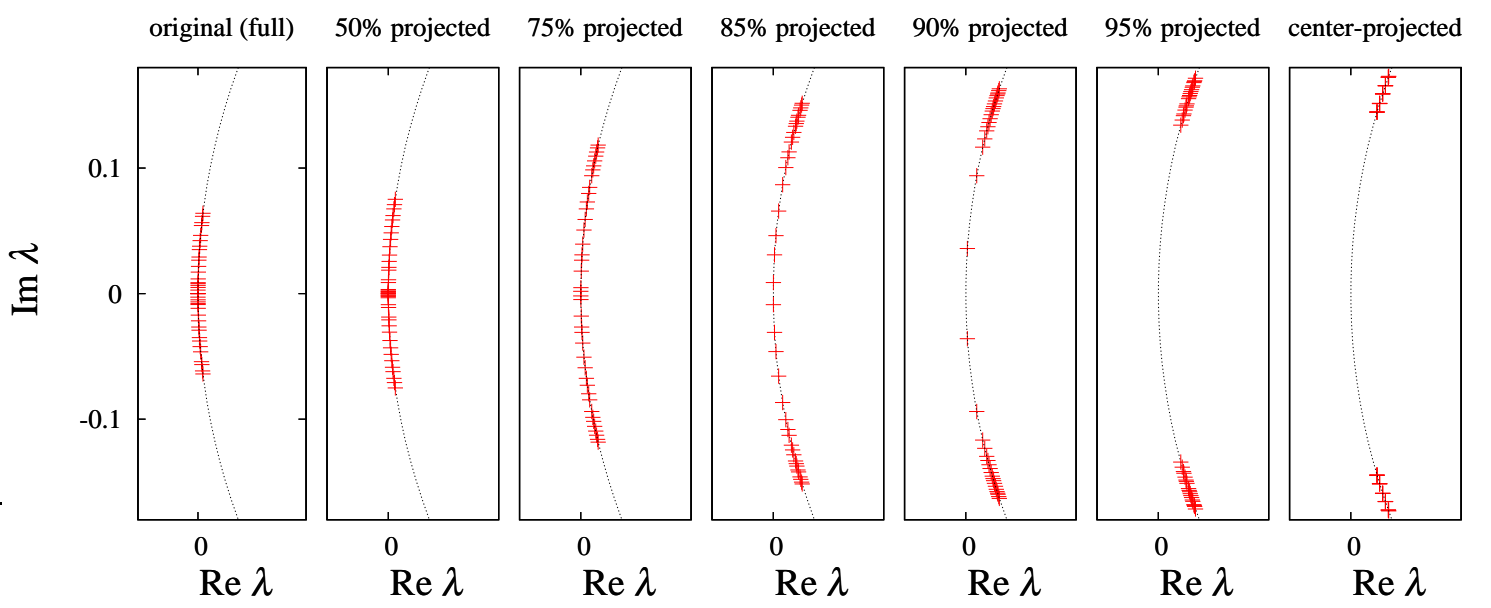

Figure 2: The first twenty overlap Dirac eigenvalue pairs from a single configuration on a $16^{4}$ lattice, antiperiodic boundary conditions at $\beta_{L W}=3.3$, for interpolated fields. 
Fig. 3 shows the first twenty asqtad eigenvalues, which distribute very differently now. The low eigenmode density (chiral condensate) increases for center-projected compared to full (original) data. Thus, for the asqtad operator, we have found exactly what was expected prior to the results of Gattnar et al.: the vortex excitations of the vortex-only lattice carry not only the information about confinement, but are also responsible for $\chi S B$ via the Banks-Casher relation.
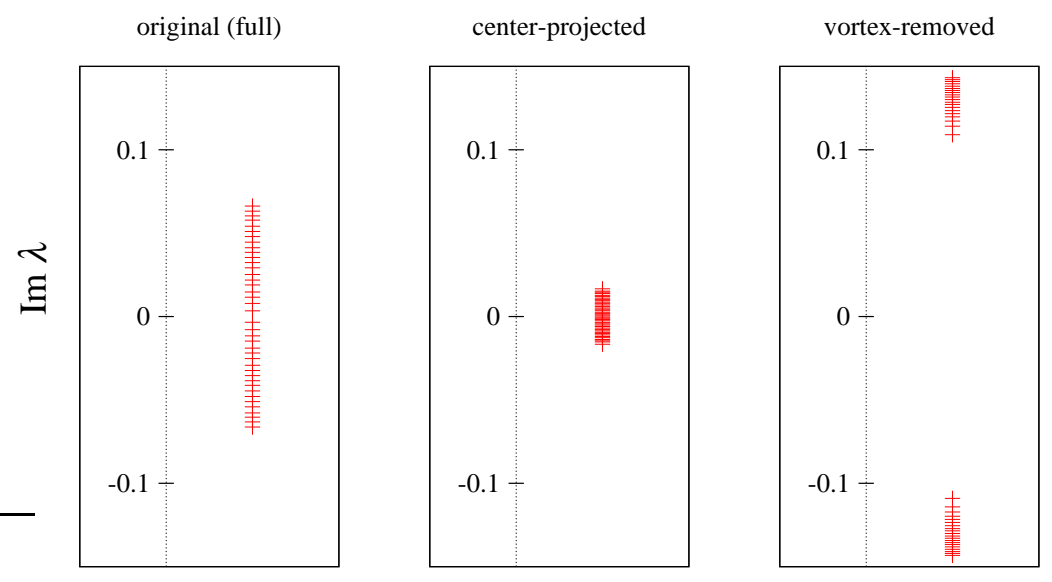

Figure 3: The first twenty asqtad Dirac eigenvalue pairs from a $16^{4}$ lattice at $\beta_{L W}=3.3$ for antiperiodic boundary conditions. The center-projected configurations show no gap around zero.

\section{Dirac eigenmode densities and vortex correlations}

The correlator $C_{\lambda}$ between the density of the eigenmode $\lambda$ and the vortex surface is investigated in order to clarify the role of the vortices in the topological structure of the vacuum. It depends on the eigenvalue and on the local geometry of the vortex. The vortex points $P_{i}$ live on the dual lattice and they are correlated to the averaged scalar eigenmode density $\rho_{\lambda}(x)$ over the 16 vertices $x$ of the $4 \mathrm{~d}$ hypercube, $H$, dual to $P_{i}$. [7]

$$
C_{\lambda}\left(N_{\mathrm{v}}\right)=\frac{\sum_{p_{i}} \sum_{x \in H}\left(V \rho_{\lambda}(x)-1\right)}{\sum_{p_{i}} \sum_{x \in H} 1}
$$

In Fig. 4 we display the data for $C_{\lambda}\left(N_{\mathrm{v}}\right)$ vs. $N_{\mathrm{v}}$ computed for eigenmodes of the asqtad Dirac operator in the full and center-projected configurations. We find that the values of $C_{\lambda}\left(N_{\mathrm{v}}\right)$ obtained from asqtad eigenmodes in the full configurations are only about a factor of four smaller than the corresponding values in the center-projected configurations. The most important feature, in our opinion, is the fact that the correlator increases steadily with increasing number of the vortex plaquettes $N_{\mathrm{v}}$, attached to a point $P_{i}$ where the Dirac eigenmode density seems to be significantly enhanced. This fact is at least compatible with the general picture advanced by Engelhardt and Reinhardt [8], that topological charge is concentrated at points where vortices either intersect, or twist about themselves ("writhe") in a certain way. 

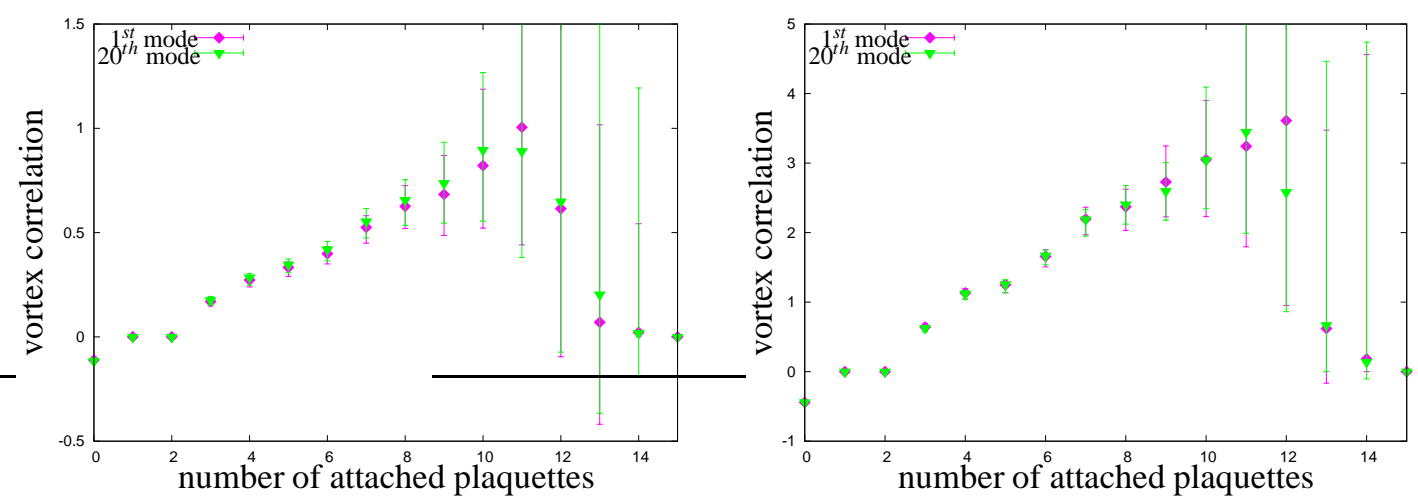

Figure 4: Vortex correlation $C_{\lambda}\left(N_{\mathrm{v}}\right)$ for asqtad staggered eigenmodes at $\beta_{L W}=3.3$ on full (left) and centerprojected (right) configurations.

\section{Conclusions}

Thin vortices found in center projection give rise to a low-lying spectrum of Dirac eigenmodes, providing that the chiral symmetry of the Dirac operator does not depend on the smoothness of the lattice configuration. Thus, the vortex excitations of the vortex-only lattice carry not only the information about confinement, but are also responsible for $\chi S B$ via the Banks-Casher relation. There are significant correlations between center vortices and the low-lying modes, supporting the picture of topological charge from center vortices. Our results indicate that center vortices have a strong effect on the existence and properties of low-lying eigenmodes of the Dirac operator. For more details see [11].

\section{References}

[1] A. Casher, Phys. Lett. B83 (1979) 395.

[2] T. Banks and A. Casher, Nucl. Phys. B169 (1980) 103.

[3] J. Gattnar et al., Nucl. Phys. B 716 (2005) 105 [arXiv:hep-lat/0412032].

[4] C. Gattringer, Phys. Rev. D 63 (2001) 114501 [arXiv:hep-lat/0003005];

C. Gattringer, I. Hip and C. B. Lang, Nucl. Phys. B 597 (2001) 451 [arXiv:hep-lat/0007042].

[5] R. Narayanan and H. Neuberger, Nucl. Phys. B443 (1995) 305 [arXiv:hep-th/9411108];

H. Neuberger, Phys. Lett. B417 (1998) 141 [arXiv:hep-lat/9707022].

[6] Kostas Orginos, Doug Toussaint and R.L. Sugar, Phys. Rev. D 60 (1999) 054503 [arXiv:hep-lat/9903032]; G.P. Lepage, Phys. Rev. D 59 (1999) 074502 [arXiv:hep-lat/9809157].

[7] A. V. Kovalenko et al. Phys. Lett. B 648 (2007) 383 [arXiv:hep-lat/0512036].

[8] M. Engelhardt, Nucl. Phys. B 585 (2000) 614 [arXiv:hep-lat/0004013];

M. Engelhardt and H. Reinhardt, Nucl. Phys. B 567 (2000) 249 [arXiv:hep-th/9907139].

[9] M. Lüscher and P. Weisz, Phys. Lett. B 158 (1985) 250.

[10] M. Lüscher, Phys. Lett. B 428 (1998) 342 [arXiv:hep-lat/9802011].

[11] R. Höllwieser, M. Faber, J. Greensite, U.M. Heller and Š. Olejník, Phys. Rev. D 78 (2008) 054508 [arXiv:hep-lat/0805.1846]. 